\title{
Effect Of Murabahah Volume Accumulation On Profitability Of Banking Institutions With Non Performing Financing (NPF) As Moderation Variables
}

\author{
Lakharis Inuzula ${ }^{1}$, Razif $^{2}$, Mia Ftriani ${ }^{2}$, Sri Wahyuni ${ }^{3}$, Yuli Asbar $^{2}$, Widyanawati Ferawati Siregar $^{4}$ \\ \{Lf.inuzula@gmail.com; razifishak@gmail.com;miafitriani@gmail.com; sri.aie.wahyuni@gmail.com; \\ joely_asbar@yahoo.com; widyana.verawaty@unimal.ac.id\} \\ ${ }^{1}$ Accounting Department, Faculty of Economics, STIE Kebangsaan, Bireuen, Indonesia. \\ ${ }^{2}$ Accounting Department, Faculty of Economics and Bussiness, Malikussaleh University, Lhokseumawe, \\ Indonesia. \\ ${ }^{3}$ Accounting Department, Faculty of Economics, STIE Lhokseumawe, Indonesia. \\ ${ }^{4}$ Management Department, Faculty of Economics and Bussiness, Malikussaleh University, Lhokseumawe, \\ Indonesia.
}

\begin{abstract}
This research was conducted to know the influence of volume of Murahabah accumulation toward profitability with non performing financing (NPF) as the moderation variable with study case at Public Islamic Banks in Indonesia from 2011-2015. The secondary data was used in this research taken from financial statements of Public Islamic Banks in 2011-2015. The method of data analysis was statistical descriptive, classical assumption test and hypothesis test. The result of hypothesis indicated that the volume of Murabahah influence positively to profitability represented by ROA. While the research result of second hypothesis indicated that non performing financing (NPF) moderated the relationship between volume of Murabahah with profitability and also NPF which became the independent variable.
\end{abstract}

Keywords: Control volume of murabahah, profitabilitas, and non performing financing.

\section{Introduction}

In 2008, Indonesia experienced a financial crisis, after the crisis of the banking sector in Indonesia has decreased, with the exception of Islamic banks still maintain its financial performance and provide comfort and security to shareholders, borrowers and depositors.

The existence of Islamic banks must be able to encourage and accelerate the process of community economic growth through Islamic banking activities, including funding and financing based on Islamic principles. Therefore, Islamic banks need to improve their performance as measured by the level of profitability produced. Return on assets (ROA) is one indicator of profitability, this is because assets are bank assets whose funds come from a large portion of public savings funds, so return on assets (ROA) is more appropriate to be used as a measure of bank profitability.

Islamic banks carry out their operational activities by offering products that are owned by the public through financing products. Murabahah financing is a mainstay of Islamic bank financing in its operational activities.

Research by (Kurniawansyah, 2017) explains that based on the current phenomenon, the principle of profit loss sharing applied by Islamic banks only dominates by $37 \%$ smaller than 
the principle of murabahah and ijarah by $63 \%$ (BI, 2013). From the Sharia Banking Statistics Data published by Bank Indonesia and the Otoritas Jasa Keuangan (OJK) 2006-2013, it was seen that murabahah financing dominated the financing channeled by Islamic Commercial Banks and Sharia Business Units. Murabahah financing up to April 2016 amounted to Rp. 117,375 billion or $58.13 \%$ of the total financing of Sharia banking in Indonesia.

Based on the current fonomena, the increase in ROA in Islamic banks was not offset by the decline in non-performing financing, only in 2012 the problematic of non-performing financing slightly decreased. While 2013 to 2015 always experienced an increase in nonperforming financing, but ROA in Sharia banking experienced instability (up and down). This can be illustrated in the diagram below.

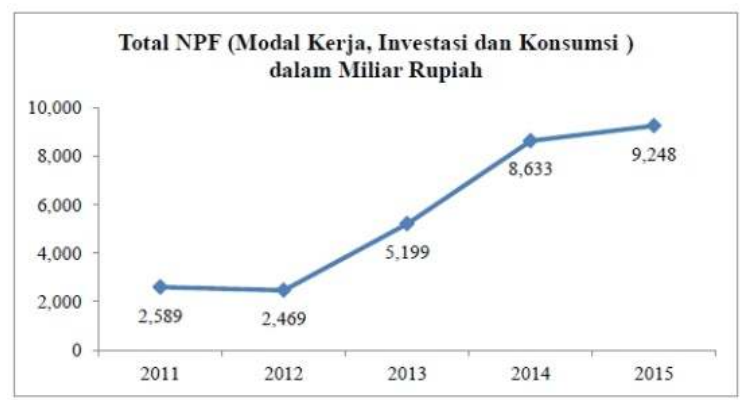

Fig. 1. TOTAL NPS BUS IN 2011 - 2015

Source: Statistik Perbankan Syariah (SPS) and OJK

Non-performing financing is a financial ratio related to credit risk. The existence of NPF can strengthen or even weaken the influence of murabahah volume on the profitability of Islamic banks, because if profitability (ROA) increases then NPF decreases and vice versa if ROA decreases then NPF has increased.

\section{Literature Review}

\subsection{Profitability}

According to (Maulana Yusuf, 2014) profitability is the end result of a policy and a decision that will be made by the company. To measure the performance of a bank can use profitability indicators. In general, profitability is projected by ROA. ROA is an indicator that can measure the profitability of a bank. The greater the ROA, the better the bank's performance. This ratio can be formulated as follows:

$$
R O A=\frac{\text { Laba Sebelum Pajak }}{\text { Total Aset }} \times 100 \%
$$

\subsection{Volume Accumulation}

According to the Kamus Besar Bahasa Indonesia (KBBI) accumulation is collection, gathering and stockpiling. Accumulation is also said to be a regular addition to a principal amount. Accumulation in Islamic banks is an additional amount of financing obtained by an Islamic bank and can generate profits or additional funds. 


\subsection{Murabahah Financing}

Murabahah is a contract of sale of goods with a sale and purchase price of the acquisition cost plus an agreed profit and the seller discloses the acquisition cost of the item to the buyer (PSAK 102 paragraph 5 in the 2016 Murabahah Sharia Banking Product Standard).

\subsection{Non-Performing Financing}

According to PSAK No. 31 (Revised 2000), stated that nonperforming financing is a loan with principal installment payments and / or interest that has exceeded 90 days or more after maturity, or credit whose payment is timely is highly doubtful. The calculation of NPF instructed by Bank Indonesia is formulated as follows:

$$
N P F=\frac{\text { Jumlah Pembiayaan Bermasalah }}{\text { Total Pembiayaan }}
$$

\section{Research Methods}

Data used in this study are secondary data based on time series obtained from Islamic Bank Financial Statements, Sharia Banking Statistics in OJK from 2011 to 2015. Secondary data are sources data obtained by researchers indirectly through intermediary media (Indriantoro, 2009, p. 50). In table 1 describe the population in this study.

Table 1. Registered Islamic Bank In Ojk

\begin{tabular}{|c|c|}
\hline \multicolumn{2}{|c|}{ Islamic Bank } \\
\hline Sharia & $\begin{array}{l}\text { Jabar Banten Sharia } \\
\text { Bank }\end{array}$ \\
\hline Sharia BNI & $\begin{array}{l}\text { Panin Dubai Sharia } \\
\text { Bank }\end{array}$ \\
\hline $\begin{array}{l}\text { Mega } \\
\text { Bank }\end{array}$ & $\begin{array}{l}\text { Bukopin } \\
\text { Bank }\end{array}$ \\
\hline $\begin{array}{l}\text { Muammalat } \\
\text { Indonesia Bank }\end{array}$ & Victoria Sharia Bank \\
\hline $\begin{array}{l}\text { Mandiri Shatia } \\
\text { Bank }\end{array}$ & BTPN Sharia Bank \\
\hline $\begin{array}{ll}\text { BCA } & \text { Sharia } \\
\text { Bank } & \\
\end{array}$ & $\begin{array}{l}\text { Maybank Indonesia } \\
\text { Sharia Bank }\end{array}$ \\
\hline $\mathrm{BRI} \mathrm{Sl}$ & \\
\hline
\end{tabular}

Table 2. Calculation Of Research Samples

\begin{tabular}{lc}
\hline \multicolumn{1}{c}{ Sample Criteria } & Calculation \\
\hline $\begin{array}{l}\text { Islamic Bank registered at OJK } \\
\text { in 2011-2015. }\end{array}$ & 13 \\
\hline $\begin{array}{l}\text { Islamic Bank that have been } \\
\text { estabilished and operate for } \\
\text { five years or more. }\end{array}$ & $(2)$ \\
\hline \multicolumn{2}{c}{ Number of Samples } \\
\hline $\begin{array}{l}\text { Number of Research } \\
\text { observations ( } 11 \text { x 5 years) }\end{array}$ \\
\hline
\end{tabular}


From 13 Islamic banks operating in Indonesia, but only 11 Islamic banks that met the criteria in this study. This is because PT Bank Aceh Syariah and PT Syariah National Pension Savings Bank have not operated for five years, thus PT Bank Aceh Syariah and PT Sharia National Pension Savings Bank do not meet the second criteria.

\subsection{Data Collection Technique}

Data collection techniques in this study use the Sharia Bank financial report, Sharia Banking Statistics (SPS) published by OJK from 2011 to 2015.

\subsection{Data Analysis Method}

Data analysis methods used in this study are descriptive statistics, classical assumption tests and hypothesis testing.

\subsection{Hypothesis Testing}

\subsubsection{Particial Significance Test}

Partially, hypothesis testing is done by t-test. If the Significance value is $<0.05$, murabahah volume accumulation variable has a significant effect on the profitability. If the Significance value is $>0.05$, the independent variable has no significant effect on the dependent variable.

\subsubsection{Moderation Regression with Interaction Test}

Interaction test or Moderated Regression Analysis (MRA) is a special application of linear multiple regression where the regression equation contains an element of interaction (multiplying two or more independent variables) with the following equation formula (Ghozali, 2002, p. 223):

\section{PROFIT $=a+b_{1} M U R A B A H A H+b_{2} N P F+b_{3} X_{1} X_{2}+$}

\section{Result And Discussion}

\subsection{General Descriptions of Sharia Banks}

Sharia banking was first present in 1963 in Mit Ghamr City based on profit sharing. In Indonesia the first commercial bank that became the pioneer of sharia banking was Muammalat Indonesia Bank, was founded in 1991, whic was initiated by the Indonesian General Assembly (MUI) and the Indonesian Government as well as the support of the Association of Ikatan Cendikiawan Muslim Indonesia (ICMI) and several Muslim entrepreneurs.

\subsection{Development of ROA, Murabahah and NPF Volume in 2011-2015}

\subsubsection{ROA Development}

Table 3. ROA Growth In $2011-2015$ (In\%)

\begin{tabular}{lclllll}
\hline $\mathrm{N}$ & Islamic & 201 & 201 & 201 & 201 & 201 \\
$\mathrm{o}$ & Bank & 1 & 2 & 3 & 4 & 5 \\
\hline
\end{tabular}




\begin{tabular}{|c|c|c|c|c|c|c|}
\hline $\mathrm{N}$ & Islamic & 201 & 201 & 201 & 201 & 201 \\
\hline o. & Bank & 1 & 2 & 3 & 4 & 5 \\
\hline \multirow[t]{2}{*}{1.} & Muammalat & 1,1 & 0,20 & 0,27 & 0,17 & 0,2 \\
\hline & $\begin{array}{c}\text { Indonesia } \\
\text { Bank }\end{array}$ & 3 & & & & \\
\hline \multirow[t]{3}{*}{2.} & Victoria & 6,9 & 1,43 & 0,50 & - & - \\
\hline & Sharia Bank & & & & 1,87 & 2,3 \\
\hline & & & & & & 6 \\
\hline \multirow[t]{2}{*}{3.} & BRI Sharia & 0,2 & 1,19 & 1,15 & 0,08 & 0,7 \\
\hline & Bank & 0 & & & & 6 \\
\hline \multirow[t]{3}{*}{4.} & Jabar & 0,6 & 0,59 & 0,91 & 0,69 & 0,2 \\
\hline & Banten & 5 & & & & 5 \\
\hline & Sharia Bank & & & & & \\
\hline \multirow[t]{2}{*}{5.} & BNI Sharia & 1,2 & 1,48 & 1,37 & 1,27 & 1,4 \\
\hline & Bank & 9 & & & & 3 \\
\hline \multirow[t]{3}{*}{6.} & Mandiri & 1,9 & 2,25 & 1,53 & - & - \\
\hline & Sharia Bank & 5 & & & 0,04 & 0,5 \\
\hline & & & & & & 6 \\
\hline \multirow[t]{2}{*}{7.} & Mega & 1,5 & 3,81 & 2,33 & 0,29 & 0,3 \\
\hline & Sharia Bank & 8 & & & & 0 \\
\hline \multirow[t]{2}{*}{8} & Panin & 2,0 & 3,48 & 1,03 & 1,99 & 1,1 \\
\hline & Sharia Bank & 6 & & & & 4 \\
\hline \multirow[t]{2}{*}{9.} & Bukopin & 0,5 & 0,55 & 0,69 & 0,27 & 0,7 \\
\hline & Sharia Bank & 2 & & & & 9 \\
\hline \multirow[t]{2}{*}{10} & BCA Sharia & 0,9 & 0,80 & 1,00 & 0,80 & 1,0 \\
\hline & Bank & 0 & & & & 0 \\
\hline \multirow{3}{*}{$\begin{array}{c}11 \\
.\end{array}$} & Maybank & 3,5 & 2,88 & 2,87 & 3,61 & - \\
\hline & Indonesia & 7 & & & & 4,7 \\
\hline & Sharia Bank & & & & & 3 \\
\hline
\end{tabular}

\subsubsection{Murabahah Volume Development}

Table 4. Murabahah Volume Development In 2011 - 2015

\begin{tabular}{|c|c|c|c|c|c|c|}
\hline $\begin{array}{l}\mathrm{N} \\
\mathrm{O}\end{array}$ & $\begin{array}{l}\text { Islamic } \\
\text { Bank }\end{array}$ & 2011 & 2012 & 2013 & 2014 & 2015 \\
\hline 1. & $\begin{array}{l}\text { Muamma } \\
\text { lat } \\
\text { Indonesia } \\
\text { Bank }\end{array}$ & 9.580 .469 .844 & 5.168 .875 .653 & 17.882 .672 .902 & $\begin{array}{l}18.375 .758 .89 \\
4\end{array}$ & 16.161 .567 .675 \\
\hline 2. & $\begin{array}{l}\text { Victoria } \\
\text { Sharia } \\
\text { Bank }\end{array}$ & 195.530 .813 & 373.964 .652 & 581.715 .763 .188 & $\begin{array}{l}479.451 .019 .8 \\
79\end{array}$ & $\begin{array}{l}360.400 .118 .99 \\
8\end{array}$ \\
\hline 3. & $\begin{array}{l}\text { BRI } \\
\text { Sharia } \\
\text { Bank } \\
\end{array}$ & $\begin{array}{l}5.369 .344 .000 . \\
000\end{array}$ & $\begin{array}{l}7.128 .905 .000 \\
.000\end{array}$ & $\begin{array}{l}9.004 .029 .000 .0 \\
00\end{array}$ & $\begin{array}{l}10.020 .738 .00 \\
0.000\end{array}$ & $\begin{array}{l}10.003 .275 .000 \\
.000\end{array}$ \\
\hline
\end{tabular}




\begin{tabular}{|c|c|c|c|c|c|c|}
\hline 4. & $\begin{array}{l}\text { Jabar } \\
\text { Banten } \\
\text { Sharia } \\
\text { Bank }\end{array}$ & 806.632 & 1.371 .919 .854 & 2.144 .892 .482 & 2.958 .627 .845 & 3.737 .449 .870 \\
\hline 5. & $\begin{array}{l}\text { BNI } \\
\text { Sharia } \\
\text { Bank } \\
\end{array}$ & $\begin{array}{l}3.113 .081 .000 . \\
000\end{array}$ & $\begin{array}{l}4.757 .648 .000 \\
.000\end{array}$ & $\begin{array}{l}7.902 .799 .000 .0 \\
00\end{array}$ & $\begin{array}{l}11.353 .027 .00 \\
0.000\end{array}$ & $\begin{array}{l}13.346 .227 .000 \\
.000\end{array}$ \\
\hline 6. & $\begin{array}{l}\text { Mandiri } \\
\text { Sharia } \\
\text { Bank }\end{array}$ & $\begin{array}{l}18.634 .180 .88 \\
5.694\end{array}$ & $\begin{array}{l}25.591 .782 .51 \\
2.796\end{array}$ & $\begin{array}{l}30.725 .052 .862 . \\
631\end{array}$ & $\begin{array}{l}30.659 .464 .90 \\
9.397\end{array}$ & $\begin{array}{l}31.577 .087 .055 \\
.815\end{array}$ \\
\hline 7. & $\begin{array}{l}\text { Mega } \\
\text { Sharia } \\
\text { Bank } \\
\end{array}$ & 3.414 .860 .147 & 5.360 .111 .256 & 6.871 .656 .664 & 5.322 .626 .134 & 4.108 .197 .905 \\
\hline 8 & $\begin{array}{l}\text { Panin } \\
\text { Sharia } \\
\text { Bank }\end{array}$ & $\begin{array}{l}382.311 .030 .0 \\
00.000\end{array}$ & $\begin{array}{l}770.966 .483 .0 \\
00.000\end{array}$ & $\begin{array}{l}1.242 .474 .076 .0 \\
00.000\end{array}$ & $\begin{array}{l}626.007 .504 .0 \\
00.000\end{array}$ & $\begin{array}{l}538.759 .169 .00 \\
0.000\end{array}$ \\
\hline 9. & $\begin{array}{l}\text { Bukopin } \\
\text { Sharia } \\
\text { Bank }\end{array}$ & $\begin{array}{l}1.280 .348 .302 . \\
715\end{array}$ & $\begin{array}{l}1.784 .352 .171 \\
.974\end{array}$ & $\begin{array}{l}2.176 .052 .977 .6 \\
08\end{array}$ & $\begin{array}{l}2.234 .996 .064 . \\
935\end{array}$ & $\begin{array}{l}2.224 .644 .728 . \\
091\end{array}$ \\
\hline 10 & $\begin{array}{l}\text { BCA } \\
\text { Sharia } \\
\text { Bank }\end{array}$ & $\begin{array}{l}330.689 .243 .3 \\
25\end{array}$ & $\begin{array}{l}431.427 .181 .0 \\
95\end{array}$ & 587.727 .197 .703 & $\begin{array}{l}910.948 .228 .4 \\
79\end{array}$ & $\begin{array}{l}1.421 .832 .070 . \\
062\end{array}$ \\
\hline 11 & $\begin{array}{l}\text { Maybank } \\
\text { Indonesia } \\
\text { Sharia } \\
\text { Bank }\end{array}$ & $\begin{array}{l}725.390 .000 .0 \\
00\end{array}$ & $\begin{array}{l}678.213 .000 .0 \\
00\end{array}$ & 733.090 .000 .000 & $\begin{array}{l}924.967 .000 .0 \\
00\end{array}$ & $\begin{array}{l}803.942 .000 .00 \\
0\end{array}$ \\
\hline
\end{tabular}

Source: financial statements for each bank

\subsubsection{Non-Performing Financing Development}

Table 5. Development Of NPF In 2011 - 2015 (In\%)

\begin{tabular}{|c|c|c|c|c|c|c|}
\hline $\begin{array}{l}\mathrm{N} \\
\mathrm{o}\end{array}$ & $\begin{array}{l}\text { Islamic } \\
\text { Bank }\end{array}$ & $\begin{array}{l}201 \\
1\end{array}$ & $\begin{array}{l}201 \\
2\end{array}$ & $\begin{array}{l}201 \\
3\end{array}$ & $\begin{array}{l}201 \\
4\end{array}$ & $\begin{array}{l}201 \\
5\end{array}$ \\
\hline 1. & $\begin{array}{l}\text { Muamma } \\
\text { lat } \\
\text { Indonesia } \\
\text { Bank }\end{array}$ & 0,01 & 0,02 & 0,02 & 0,06 & $\begin{array}{l}0,0 \\
8\end{array}$ \\
\hline 2. & $\begin{array}{l}\text { Victoria } \\
\text { Sharia } \\
\text { Bank }\end{array}$ & 0,19 & 0,11 & 0,05 & 0,13 & $\begin{array}{l}0,2 \\
3\end{array}$ \\
\hline 3. & $\begin{array}{l}\text { BRI } \\
\text { Sharia } \\
\text { Bank }\end{array}$ & 0,04 & 0,04 & 0,04 & 0,04 & $\begin{array}{l}0,0 \\
5\end{array}$ \\
\hline 4. & $\begin{array}{l}\text { Jabar } \\
\text { Banten } \\
\text { Sharia } \\
\text { Bank }\end{array}$ & 0,03 & 0,07 & 0,01 & 0,05 & $\begin{array}{l}0,0 \\
4\end{array}$ \\
\hline 5. & $\begin{array}{l}\text { BNI } \\
\text { Sharia }\end{array}$ & 0,02 & 0,02 & 0,02 & 0,02 & $\begin{array}{l}0,0 \\
2\end{array}$ \\
\hline
\end{tabular}




\begin{tabular}{|c|c|c|c|c|c|c|}
\hline $\mathrm{N}$ & Islamic & 201 & 201 & 201 & 201 & 201 \\
\hline o & Bank & 1 & 2 & 3 & 4 & 5 \\
\hline & Bank & & & & & \\
\hline 6. & $\begin{array}{l}\text { Mandiri } \\
\text { Sharia } \\
\text { Bank }\end{array}$ & 0,03 & 0,03 & 0,05 & 0,07 & $\begin{array}{l}0,0 \\
6\end{array}$ \\
\hline 7. & $\begin{array}{l}\text { Mega } \\
\text { Sharia } \\
\text { Bank }\end{array}$ & 0,03 & 0,03 & 0,03 & 0,03 & $\begin{array}{l}0,0 \\
4\end{array}$ \\
\hline 8 & $\begin{array}{l}\text { Panin } \\
\text { Sharia } \\
\text { Bank } \\
\end{array}$ & 0,02 & 0,00 & 0,02 & 0,02 & $\begin{array}{l}0,0 \\
5\end{array}$ \\
\hline 9. & $\begin{array}{l}\text { Bukopin } \\
\text { Sharia } \\
\text { Bank } \\
\end{array}$ & 0,01 & 0,05 & 0,04 & 0,04 & $\begin{array}{l}0,0 \\
3\end{array}$ \\
\hline $\begin{array}{l}1 \\
0 .\end{array}$ & $\begin{array}{l}\text { BCA } \\
\text { Sharia } \\
\text { Bank } \\
\end{array}$ & 0,00 & 0,00 & 0,00 & 0,00 & $\begin{array}{l}0,0 \\
1\end{array}$ \\
\hline $\begin{array}{l}1 \\
1 .\end{array}$ & $\begin{array}{l}\text { Maybank } \\
\text { Indonesia } \\
\text { Sharia } \\
\text { Bank }\end{array}$ & 0,01 & 0,02 & 0,02 & 0,00 & $\begin{array}{l}0,3 \\
6\end{array}$ \\
\hline
\end{tabular}

\subsection{Data Quality Test}

\subsubsection{Descriptive Statistics}

Table 6. Descriptive Statistics Results

\begin{tabular}{|c|c|c|c|c|}
\hline \multicolumn{5}{|c|}{ log_volumlog_nplog_profitablog_moderas } \\
\hline & $\begin{array}{c}\text { e_murabo } \\
\text { hah }\end{array}$ & $\mathrm{f}$ & ilitas & $\overline{\mathrm{ix}} 1 \times 2$ \\
\hline $\begin{array}{c}\mathrm{N} \\
\text { Valid } \\
\end{array}$ & 55 & 49 & 51 & 49 \\
\hline $\begin{array}{c}\text { Missin } \\
\mathrm{g}\end{array}$ & 0 & 6 & 4 & 6 \\
\hline Mean & 11.7182 & $\begin{array}{c}- \\
1.4574 \\
\end{array}$ & -.0395 & -17.0551 \\
\hline $\begin{array}{c}\text { Std. } \\
\text { Deviati } \\
\text { om } \\
\end{array}$ & 1.86985 & .34549 & .40879 & 5.06708 \\
\hline $\begin{array}{l}\text { Minim } \\
\text { um }\end{array}$ & 5.91 & -2.00 & -1.10 & -25.64 \\
\hline $\begin{array}{c}\text { Maxim } \\
\text { um }\end{array}$ & 15.09 & -.44 & .84 & -5.28 \\
\hline Sum & 644.50 & -71.41 & -2.01 & -835.70 \\
\hline
\end{tabular}

Source: Secondary data processed by SPSS 17.0 (2017)

Based on table 6. shows that ROA of Islamic banking in Indonesia is categorized as not good. This is based on Bank Indonesia regulation which explains that ROA of Islamic banking 
can be categorized as good if it has a value above $1.5 \%$. Whereas the volume of murabahah distributed by Islamic banks has a good rate of return. This is in accordance with Bank Indonesia regulations which require NPF to be below 5\%.

\subsubsection{Clasic Assumption Test}

Normality test can be seen by using the Kolmogrov Smirnov (K-S) test and the normal probability plot. Based on the results of the normality test, it can be seen that the data is normally distributed.

\subsection{Hypothesis Testing}

\subsubsection{First Hypothesis Testing (H1)}

The $t$ test in this study was namely Profitability proxied by ROA during the period 20112015. The results of this study are also the same as the results of research from (Afif and Mawardi, 2015; Rizwanullah, 2015) whose results state that murabahah financing affects profitability. murabahah financing is a financing that is the mainstay of Islamic commercial banks in generating profits that will affect the level of profitability and will affect the growth rate of sharia commercial banks in Indonesia.

\subsubsection{Second Hypothesis Testing (H2)}

Moderation Regression with Interaction Test or Moderated Regression Analysis (MRA) Test. In this study the moderating variable is also called the second independent variable. In carrying out this test that must be considered is the coefficient of determination, simultaneous significance test and partial significance test.

a. Determination Coefficient

The coefficient of determination is the comparison between variation $\mathrm{Y}$ described by X1 and $\mathrm{X} 2$ together compared to total variation $\mathrm{Y}$ or this can also be interpreted as how much ability explain all.

b. Simultaneous Significance Test

Based on the ANOVA test or F test produces Fcount value of 3.205 with a significance level of 0.033 , cannot be used to predict $Y$ or it can be said that the volume of murabahah, NPF and moderationX1X2 together influence against profitability. So that it can be said that the volume of murabahah, non-performing financing (NPF) and moderation X1X2 (multiplication between $\mathrm{X} 1$ and $\mathrm{X} 2$ ) together affect the profitability that is proxied by ROA.

c. Partial Significance Test

The murabahah volume variable gives the parameter coefficient of -0.436 with a significance level of 0.014 or $0.014<0.05$. The NPF variable gives a parameter coefficient of 3.027 with a significance level of 0.017 or $0.017<0.05$. The moderation variable X1X2 gives a parameter coefficient of -0.308 with a significance level of $0.009(<0.05)$. Moderation variable X1X2 which is an interaction between the volume of murabahah and non-performing financing (NPF) turns out to be significant, so it can be concluded that non-performing financing (NPF) is a moderating variable.

In this case, the second hypothesis or $\mathrm{H} 2$ is accepted, because NPF moderates (strengthens) the relationship between murabahah volume and profitability and also nonperforming financing (NPF) as well as being an independent variable. 
The results of this study are the same as the results of Septian (Maulana Yusuf, 2014; Wahyuni, 2016) whose research results state that the simultaneous problematic financing of murabahah financing has a very strong relationship and has a significant effect on profitability

\section{Conclusions}

Based on the discussion that has been described, it can be concluded as follows:

1. The test results for the first hypothesis (H1) show that murabahah volume has a significant effect on profitability proxied by ROA.

2. Test results for the second hypothesis $(\mathrm{H} 2)$ indicate that:

a) Murabahah volume, NPF and moderation X1X2 together affect the profitability that is traded with ROA.

b) Partial significance test shows that NPF moderates (strengthens) the relationship between murabahah volume and profitability and NPF as well as being an independent variable.

Based on the conclusions of the study, the authors recommend the following suggestions: This research is not comprehensive, because it only sees murabaha included in the category of receivables as independent variables. For those who want to do the next research so that they can add greetings, istishna and ijarah which are also included in the category of receivables included in the category of receivables in addition to independent variables.

\section{References}

[1] Afif, Z. N. and Mawardi, I. (2015) 'Pengaruh Pembiayaan Murabahah Terhadap Laba Melalui Variabel Intervening Pembiayaan Bermasalah Bank Umum Syariah di Indonesia Periode 2009-2013', Jurnal Ekonomi Syariah Teori dan Terapan, 1(8). doi: 10.20473/VOL1ISS20148PP\%P.

[2] Ghozali, I. (2002) Application of Multivariate Analysis with SPSS program. Semarang: National Publishers Diponegoro University.

[3] Indriantoro, N. B. S. (2009) Metodelogi Penelitian Bisnis Untuk Akuntansi Dan Manajemen. Edisi Pertama. Yogyakarta: BPFE Yogyakarta.

[4] Kurniawansyah, D. (2017) 'Pengaruh Profit Loss Sharing Funding Ratio dan Profit Loss Sharing Financing Ratio Terhadap Profitabilitas Bank Umum Syari'ah di Indonesia dengan Efisiensi dan Risiko Sebagai Variabel Intervening', Jurnal Akuntansi dan Keuangan, 18(1), pp. 44-58. doi: 10.9744/JAK.18.1.44-58.

[5] Maulana Yusuf, S. (2014) Kredit Bermasalah Pembiayaan Murabahah dan Pembiayaan Mudharabah terhadap Profitabilitas dengan Menggunakan Pendekatan Return On Assets (ROA) Studi Kasus pada PT. Bank Syariah Mandiri. Universitas Komputer Indonesia. Available at: https://repository.unikom.ac.id/28185/ (Accessed: 24 January 2019).

[6] Rizwanullah, M. I. (2015) Analisis Pembiayaan Murabahah dan Tingkat Risiko Pembiayaan (NPF) Terhadap Profitabilitas (Studi pada Bank Umum Syariah yang Terdaftar di Otoritas Jasa Keuangan). Universitas Komputer Indonesia. Available at: https://elib.unikom.ac.id/files/disk1/699/jbptunikompp-gdl-muhamadirs-34939-1unikom_m-l.pdf (Accessed: 24 January 2019).

[7] Wahyuni, M. (2016) 'Pengaruh Volume Pembiayaan Bagi Hasil dan Pembiayaan Murabahah terhadap Kinerja Keuangan Bank Umum Syariah dengan NPF sebagai Variabel Moderasi', Jurnal EBBANK, 7(1), pp. 1-10. Available at: 
http://ebbank.stiebbank.ac.id/index.php/EBBANK/article/viewFile/84/85

24 January 2019).

(Accessed: 